\title{
A NOTE ON SUM-FREE SETS OF INTEGERS
}

\author{
MEN-CHANG HU
}

\begin{abstract}
A set $S$ of integers is said to be sum-free if $a, b \in S$ implies $a+b \notin S$. Let $g(n, k)$ denote the cardinality of a largest subset of $\{1,2, \ldots, n\}$ that can be partitioned into $k$ sum-free sets. In this note we show that $g(n, 2)=n-[n / 5]$.
\end{abstract}

Let $k$ be an integer. A set $S$ of integers is said to be sum-free modulo $k$ if $a$, $b \in S$ implies $a+b \equiv x(\bmod k)$ has no solution in $S$. A set which is sum-free modulo 0 is customarily called sum-free. Let $g(n, k)$ denote the cardinality of a largest subset of $\{1,2, \ldots, n\}$ that can be partitioned into $k$ sum-free subsets, and let $h(k)$ denote the largest integer $m$ for which there exists some way of partitioning $\{1,2, \ldots, m\}$ into $k$ subsets which are sum-free modulo $m+1$.

The numbers $g(n, k)$ and $h(k)$ were introduced in [1], where H. L. Abbott and E. T. H. Wang proved, among other things, that

(1) $g(n, k)>n-[n /(h(k)+1)]$,

(2) $g(n, 1)=n-[n /(h(1)+1)]=[(n+1) / 2]$,

(3) $g(n, 2)=n-[n /(h(2)+1)]=n-[n / 5]$ for $n<54$.

They also conjectured that the equality sign holds in (1). In this note we shall prove this conjecture for the case $k=2$.

THEOREM. $g(n, 2)=n-[n / 5]$.

Proof. A proof of $g(n, 2)>n-[n / 5]$ was given in [1]. We include it here for completeness. For an arbitrary fixed positive integer $n$, let

$$
\begin{aligned}
& N=\{1,2, \ldots, n\}, \quad A=\{p \in N: p \equiv 1 \text { or } 4(\bmod 5)\}, \\
& B=\{p \in N: p \equiv 2 \text { or } 3(\bmod 5)\} .
\end{aligned}
$$

If $a, b \in A$, then $a+b \equiv 0,2$ or $3(\bmod 5)$. This implies $a+b \notin A$. Thus $A$ is sum-free. Similarly, $B$ is sum-free. since $A \cup B$ has $n-[n / 5]$ elements, $g(n, 2)>n$ $-[n / 5]$ by definition.

Now we show that $g(n, 2)<n-[n / 5]$. Assume that $S \subset N$ can be partitioned into two sum-free subsets $S_{1}$ and $S_{2}$. Let $R=N \backslash S$. Write $n=5 q+j, 0<j<4$. Our aim is to show that $R$ contains at least $q$ elements. We may assume that $S$ has no less than $4 q+j+1$ elements. Then either $S_{1}$ or $S_{2}$ has at least $2 q+1$ elements. Without loss of generality, we assume that $S_{1} \supset\left\{x_{0}<x_{1}<\cdots<x_{2 q}\right\}$. Let $D=\left\{x_{r}-x_{0}: r=1, \ldots, 2 q\right\}$. Clearly $D \subset N$ and $D$ has $2 q$ elements. Since

Received by the editors October 31, 1979.

1980 Mathematics Subject Classification. Primary 05A17.

Key words and phrases. Sum-free set. 
$S_{1}$ is sum-free $D \cap S_{1}=\varnothing$. So $D \subset S_{2} \cup R$. If $D \cap R$ has no less than $q$ elements we are done. So we assume that $D \cap R$ contains less than $q$ elements, then $D \cap S_{2} \supset\left\{y_{0}<y_{1}<\cdots<y_{q}\right\}$, where, for each $s$ with $0<s<q, y_{s}=x_{r_{s}}$ $-x_{0}$ for some $r_{s}$ with $1<r_{s}<2 q$. Let $z_{t}=y_{t}-y_{0}, t=1, \ldots, q$. Then $z_{t} \in N$ and $z_{t_{1}}<z_{t_{2}}$ if $t_{1}<t_{2}$. Since $S_{2}$ is sum-free, $z_{t} \notin S_{2}$. Also

$$
z_{t}=\left(x_{r_{t}}-x_{0}\right)-\left(x_{r_{0}}-x_{0}\right)=x_{r_{t}}-x_{r_{0}} \notin S_{1} \text {. }
$$

So $z_{t} \in R$ for all $t=1, \ldots, q$, and hence $R$ has at least $q$ elements. This completes the proof.

\section{REFERENCES}

1. H. L. Abbott and E. T. H. Wang, Sum-free sets of integers, Proc. Amer. Math. Soc. 67 (1977), 11-16.

Instrtute of Mathematics, Academa Sinica, Taipei, Taiwan, R.O.C. 